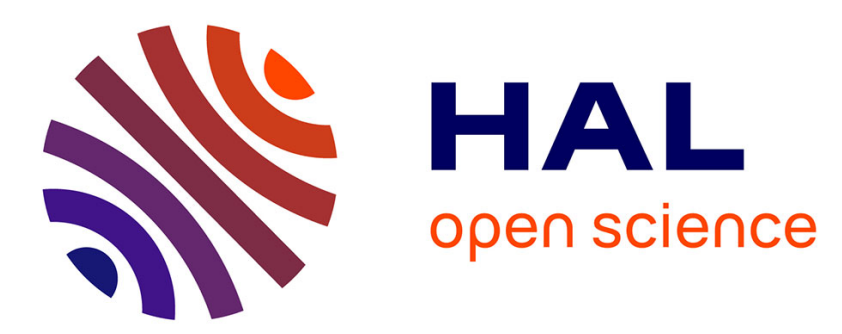

\title{
Mise en évidence du Tomato Black Ring Virus (TBRV) chez l'artichaut en France
}

Auguste Migliori, Hervé Marzin, Gian Luigi Rana, Véronique Legal, Etienne Homo

\section{- To cite this version:}

Auguste Migliori, Hervé Marzin, Gian Luigi Rana, Véronique Legal, Etienne Homo. Mise en évidence du Tomato Black Ring Virus (TBRV) chez l'artichaut en France. Agronomie, 1984, 4 (7), pp.683-686. hal-00884686

\section{HAL Id: hal-00884686 https://hal.science/hal-00884686}

Submitted on 1 Jan 1984

HAL is a multi-disciplinary open access archive for the deposit and dissemination of scientific research documents, whether they are published or not. The documents may come from teaching and research institutions in France or abroad, or from public or private research centers.
L'archive ouverte pluridisciplinaire HAL, est destinée au dépôt et à la diffusion de documents scientifiques de niveau recherche, publiés ou non, émanant des établissements d'enseignement et de recherche français ou étrangers, des laboratoires publics ou privés. 


\title{
Mise en évidence du Tomato Black Ring Virus (TBRV) chez l'artichaut en France
}

\author{
Auguste MIGLIORI, Hervé MARZIN (*), Gian Luigi RANA (**) \\ avec la collaboration technique de Véronique LEGAL et Etienne Homo \\ I.N.R.A., Station de Pathologie végétale, Centre de Recherches de Rennes, F 35650, Le Rheu (I) \\ (*) Service de la Protection des Végétaux, G.R.I.S.P. de Rennes, Domaine de la Motte, F 35650, Le Rheu \\ (**) Universita degli studi di Bari, Dipartimento di Patologia vegetale, via G. Amendola 165/A, I 70126 Bari
}

Le Tomato Black Ring Virus (TBRV) a été isolé en France de l'artichaut (Cynara scolymus L.) et de Veronica persica Poir. (Scrophulariacées) naturellement infectés.

L'isolat artichaut, dénommé TBRV-A, a été identifié au TBRV d'après les réactions des plantes-hôtes et les propriétés sérologiques. La transmission du virus au niveau du sol, la présence du nématode Longidorus attenuatus Hooper dans la rhizosphère des plantes infectées et la contamination de C. scolvmus, Nicotiana tabacum L. var. "Xanthi n. c. ", Physalis floridana Rydb. par L. attenuatus, ont été mises en évidence.

Mots clés additionnels : Cynara scolymus, Longidorus attenuatus, népovirus, sérologie, transmission.

TBRV was isolated in France from naturally infected artichoke (Cynara scolymus L.) and Veronica persica Poir. (Scrophulariaceae). The identification was based on the host-plant reactions and serological properties of the isolate known as TBRV-A. The virus has been shown to be soil-transmitted and Longidorus attenuatus Hooper has been shown to be present in the rhizosphere of infected plants. This nematode has been shown to transmit the virus to C. scolymus, Nicotiana tabacum L. Xanthi n.c. and Physalis floridana Rydb.

Additional key words : Cynara scolymus, Longidorus attenuatus, nepovirus, serology, transmission.

\section{INTRODUCTION}

L'étude actuellement en cours sur les virus de l'artichaut a permis de révéler un isolat différent des 3 virus déjà décrits et s'identifiant à l'Artichoke Latent Virus (ALV), au Broad Bean Wilt Virus (BBWV) et au Cucumber Mosaic Virus (CMV), (MIGLIORI et al., 1984).

Ce virus isolé des cultures d'artichaut de Bretagne, non répertorié chez le genre Cynara par RANA \& MARTELLI (1983), regroupe les caractéristiques du népovirus Tomato Black Ring Virus (TBRV) décrit par MURANT (1970).

Cette note propose l'identification de ce $4^{e}$ isolat artichaut basée sur les propriétés sérologiques, les réactions

(1) Cette étude réalisée en Bretagne s'intègre dans le programme pluridisciplinaire de la « Fatigues de sols » en culture d'artichaut soutenu par l'E.P.R. de Bretagne. des hôtes différentiels et la transmission du virus par le nématode Longidorus attenuatus Hooper.

\section{MATÉRIEL ET MÉTHODES}

Le virus est isolé d'artichaut var. «Camus de Bretagne » naturellement infecté et de Veronica persica Poir.

\section{A. Transmission du virus}

\section{Par voie mécanique}

Les transmissions par voie mécanique sont effectuées en présence de charbon végétal et de carborundum $(80 \mathrm{mg} / \mathrm{ml}$ dans les 2 cas) après broyage des feuilles infectées dans un tampon phosphate $0,01 \mathrm{M}-\mathrm{pH} 7$, contenant 0,2 p. 100 de DIECA dans le rapport $1 \mathrm{~g} / 4 \mathrm{~m} l$. 


\section{Au niveau du sol}

La terre prélevée dans la rhizosphère de la plante naturellement infectée est placée dans une terrine dans laquelle sont repiqués de jeunes plants de Nicotiana tabacum L. var. «Xanthi n. c. » et de Physalis floridana Rydb (plantes-pièges).

Dans d'autres expériences, des débris de racines d'artichaut infecté sont introduits volontairement dans le substrat non infesté par $L$. attenuatus avant le repiquage des plantules saines.

\section{A partir de L. attenuatus}

Les $L$. attenuatus prélevés aux pieds d'artichauts infectés sont groupés par lot de 30 et broyés à l'aide d'un verre de montre dans une goutte de tampon phosphate $0,01 \mathrm{M} \mathrm{--}$ pH 7. Le broyat est inoculé en présence de carborundum à $N$. tabacum Xanthi et à $P$. floridana.

\section{Par L. attenuatus}

Les $L$. attenuatus prélevés dans la rhizosphère des plantes atteintes sont déposés au niveau des racines de jeunes $P$. floridana semés dans un mélange stérile de sable et terre dans le rapport $\mathrm{V} / \mathrm{V}$.

\section{B. Extraction des nématodes}

La terre infestée est placée sur un tamis de $2 \mathrm{~mm}$, puis filtrée avec apport d'eau, le filtrat récupéré dans un seau est mélangé, puis mis au repos 2 à $5 \mathrm{mn}$. Le surnageant est filtré sur tamis de $170 \mu$, l'opération est répétée 3 fois. Le tamis est ensuite placé sur une coupelle contenant de l'eau. Celui-ci est surélevé légèrement $(2 \mathrm{~mm})$ de façon à permettre aux nématodes de migrer à travers les mailles. La récupération des nématodes est immédiate en vue de leur utilisation pour les différents tests.

\section{Sérologie}

Les épreuves sérologiques de double immunodiffusion en gélose sont réalisées en agarose (Indubiose A 37) à 0,8 p. 100 dans un tampon veronal de $\mathrm{pH} 8,4$. Les immunsérums utilisés sont : Broad Bean Wilt Virus (BBWV), (Sérothèque du département de Pathologie végétale, Université de Bari - Italie), Cucumber Mosaic Virus (CMV), Tomato Black Ring Virus, souche Bouquet Allemande (TBRV-BU 571) et Ecossaise (TBRV-W 661) obtenus par DEVERGNE \& CARDIN (Station de Pathologie végétale, INRA - Antibes) et artichaut (TBRV-A), (RANA et al., 1985).

\section{RÉSULTATS}

\section{A. Propriétés biologiques}

\section{Symptomatologie sur plantes en culture}

Les plantes atteintes par ce virus n'extériorisent pas de symptômes caractéristiques. La plante peut cepen- dant avoir un développement général plus faible notamment au démarrage de la végétation.

\section{Réactions des hôtes secondaires}

Le virus se transmet facilement par voie mécanique et infecte de nombreuses plantes herbacées. Les réactions les plus caractéristiques sont celles de : Beta vulgaris L., anneaux chlorotiques ; N. tabacum « Xanthi n.c. ", lésions locales nécrotiques ou anneaux nécrotiques et réaction systémique formée par des anneaux et mosaïque nécrotiques (Pl. 1, fig. 1). Cucurbita pepo Duch. var. «F1 Diamant », lésions locales chloro-nécrotiques et mosaïques; Vigna unguiculata (L.) Walp. ( $V$. sinensis), lésions locales ou anneaux nécrotiques, mosaïque nécrotique ou nécroses généralisées.

Une variabilité des réactions est observée chez les hôtes avec cet isolat initialement transmis par $L$. attenuatus, notamment si l'inoculum est par la suite obtenu de $C$. pepo et comparé à celui de $N$. tabacum (tabl. 1).

\section{Transmission du virus}

\section{a) Transmission au niveau du sol}

Les plantes-pièges saines sont contaminées après repiquage dans la terre naturellement infestée par $L$. attenuatus et prélevée aux pieds d'artichauts infectés. Le taux de contamination peut être très élevé, jusqu'à 20 plants sur 47 avec $P$. floridana.

La contamination des plantes-pièges n'est pas observée avec un sol prélevé dans la rhizosphère de plantes infectées mais sans la présence de $L$. attenuatus. De même, la transmission du virus n'est pas assurée en l'absence du nématode, malgré la présence dans le substrat de débris organiques contaminés.

\section{b) Transmission à partir d'extraits de nématodes}

A plusieurs reprises, le broyat de $30 \mathrm{~L}$. attenuatus prélevés aux pieds de plantes infectées a induit une réaction virale chez les plantes-pièges après inoculation.

\section{c) Transmission par L. attenuatus}

La contamination de $C$. scolymus, lignée CAVO 1-2-4 et CECU ainsi que de $P$. floridana a été obtenue par des $L$. attenuatus prélevés directement aux pieds de plants atteints et placés au niveau des racines des jeunes plantules saines maintenues dans un substrat stérilisé.

\section{B. Propriétés sérologiques}

Des arcs de précipitations sont obtenus avec l'antigène artichaut (TBRV-A) et les immunsérums TBR V-A, TBRV, BU 571 et TBRV-W 661 (Pl. 1, fig. 2, 3, 4).

Les épreuves sérologiques effectuées avec les antigènes suivants :

Antigène (a) : TBRV-A transmis d'artichaut à $P$. floridana mécaniquement. 

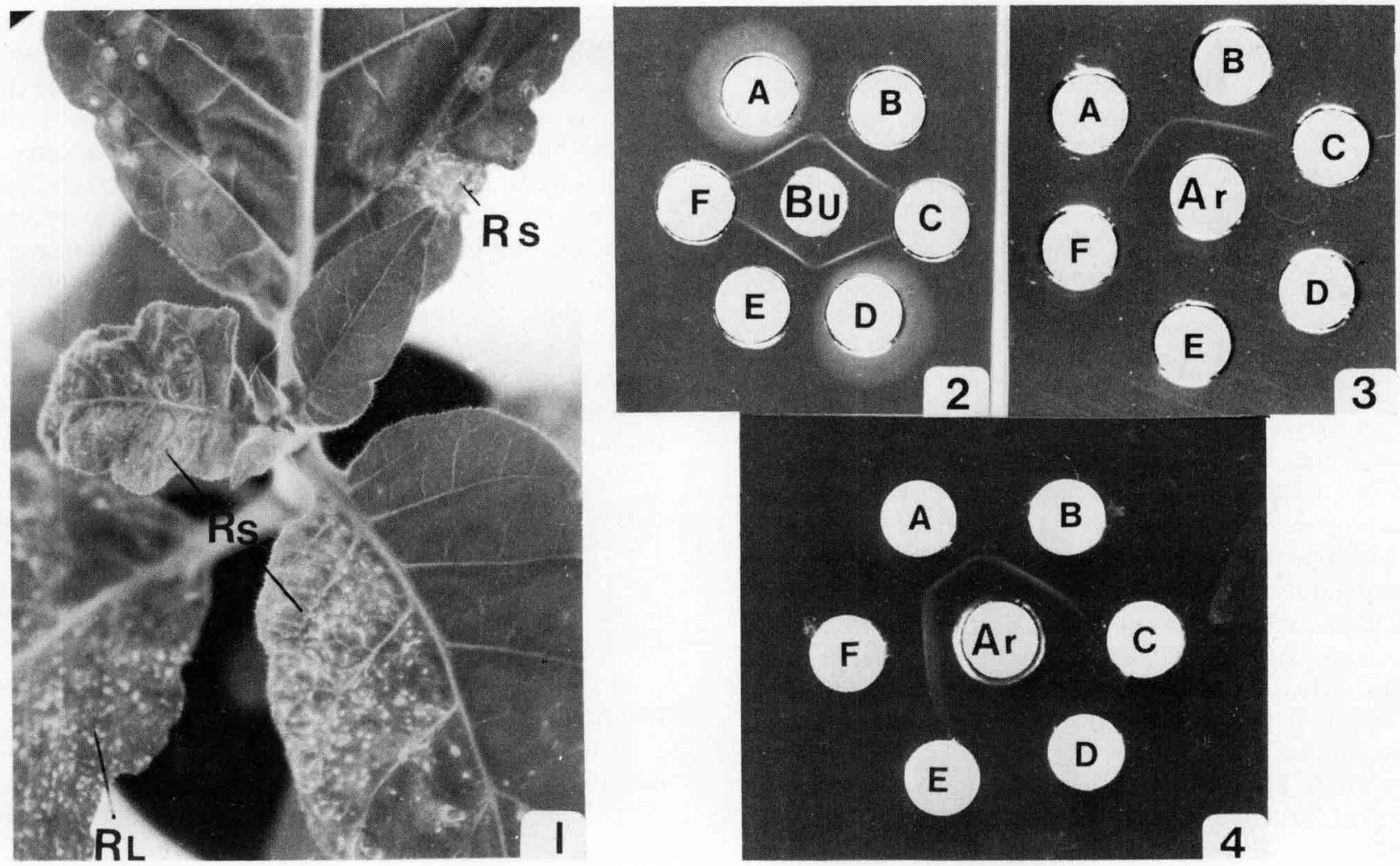

Figure 1

Nicotiana tabacum' Xanthi n.c. infecté par l'isolat artichaut (TBRV-A). Réaction locale (RL), Réaction systémique (Rs.). N. tabacum Xanthi infected by artichoke isolate (TBRV-A). Local reaction (RL). Systemic reaction (Rs).

Figures 2, 3, 4

Réactions sérologiques en immunodiffusion.

Serological reactions obtained in immunodiffusion.

Figure 2

Antisérums TBRV-BU $57 I$ (Bu), Antigènes TBRV-A transmis mécaniquement d'artichaut à $\mathrm{P}$. floridana $(A), T B R V$ - $A$ transmis au niveau du sol à $\mathrm{P}$. floridana $(B)$, Témoin $\mathrm{P}$. floridana plante saine $(C), T B R V$ - $A$ transmis à $\mathrm{P}$. floridana à partir de broyat de $\mathrm{L}$. attenuatus $(D)$, $T B R V-A$ transmis $\grave{a} \mathrm{P}$. floridana par $\mathrm{L}$. attenuatus $(E), B B W V$ isolat artichaut $(F)$.

Figure 3

Antisérums TBRV-W661 (A), TBRV-A (B), BBWV-I (C), CMV (D), Témoin sain (E), BBWV-2I (F), Antigène TBRV-A (Ar).

Figure 4

Antisérums TBRV-A (A), TBRV-BU 571 (B), BBWV-I (c), CMV (D), BBWV-2I (E), TBRV-W 661 (F), Antigène TBRV-A (Ar).

TABLEAU 1

Variabilité des réactions

Variability of responses

\begin{tabular}{|c|c|c|}
\hline \multirow[b]{2}{*}{ Plantes-hôtes } & \multicolumn{2}{|c|}{ Isolat Artichaut } \\
\hline & Inoculum C. pepo & $\begin{array}{c}\text { Inoculum } N . \text { tabacum } \\
\text { «Xanthi } »\end{array}$ \\
\hline Chenopodium quinoa & $\mathrm{LLc} / \mathrm{D}, \mathrm{TN}$ & LLn/NI \\
\hline C. pepo & $\mathrm{LLc}, \mathrm{n} / \mathrm{Mo}$ & $\operatorname{LLn} / \mathrm{NI}$ \\
\hline N. tabacum «Xanthi » & LLn, An n/An c, Re & LLn, An n/An n, Mon \\
\hline Ph. floridana & $\mathrm{Ni} / \mathrm{Mo}$ & LLn/Mon \\
\hline
\end{tabular}

Réaction locale/systémique - Local/systemic symptoms.

$\begin{array}{lllll}\text { An }: \text { Anneau } & \text { Ringspot } & \mathrm{n}: \text { Nécrotique } & \text { Necrotic } \\ \mathrm{c}: \text { Chlorotique } & \text { Chlorotic } & \mathrm{Re}: \text { Rétablissement } & \text { Recovery } \\ \mathrm{D} & \text { : Distorsion } & \text { Distorsion } & \mathrm{TN}: \text { Nécrose apicale } & \text { Top necrosis } \\ \mathrm{LL} & \text { : Lésion locale } & \text { Local lesion } & \mathrm{Ni}: \text { : Non infecté } & \text { Not infected }\end{array}$

Mo : Mosaïque Mosaic 
TBRV-A transmis au niveau du sol à $P$. floridana.

TBRV-A transmis à $P$. floridana à partir de broyat de L. attenuatus.

TBRV-A transmis à $P$. floridana par $L$. attenuatus, ont conduit aux mêmes résultats.

Aucune réaction n'a été observée avec les immunsérums BBWV et CMV.

\section{DISCUSSION}

Un virus transmis par $L$. attenuatus, nématode mentionné par DALMASSO \& CAUBEL (1966), DALMASSO (1970), a été isolé de l'artichaut et de $V$. persica (Scrophulariacées).

Les réactions des hôtes différentiels, les épreuves sérologiques et la transmission de l'isolat au niveau du sol par L. attenuatus ont permis de différencier ce virus des ALV, BBWV et CMV mentionnés aussi chez l'artichaut (MiglioRI et al., 1984) et conduisent à l'identification de ce virus au Tomato Black Ring Virus que nous dénommons TBR V-A.

L'étude biochimique, cytologique et sérologique de l'isolat TBRV-A comparée à celle des pathotypes TBRV et népovirus, réalisée en collaboration avec G. L. RANA, fera l'objet d'une publication ultérieure.

Le TBRV est mis en évidence pour la $1^{\text {re }}$ fois chez le genre Cynara. Ce népovirus a, par contre, été signalé dans l'ouest sur laitue et primevère (MORAND \& POUTIER, 1978).

L'étude en cours concernant la distribution des virus chez l'artichaut en Bretagne n'est pas en mesure de révéler à ce jour la répartition du TBRV-A.

L'étude comparative du TBRV-A aux principaux pathotypes : Beet ringspot strain, Celery yellow vein strain, Lettuce rinspot strain, Potato bouquet strain of Köhler, Potato pseudo-aucuba strain of Köhler mentionnés par MURANT (1970) sera entreprise afin de situer le TBRV-A tant sur le plan de la pathogénie que sur celui de la capacité vectrice des différentes espèces de Longidorus notamment $L$. attenuatus et caespiticola observés en Bretagne, ou L. macrosoma, vecteur connu du TBRV-E (souche anglaise), (HARRISON et al., 1961).

Une étude préliminaire avec TBRV-A confirme une capacité vectrice de $L$. attenuatus très élevée et une variabilité des réactions chez les hôtes. En ce sens, il serait important de déterminer si ces caractéristiques sont bio-génétiques et auto-modifiables dans le trinôme Pathogène-Hôte-Vecteur ou s'il s'agit d'une simple spécificité pathogénique. La même question pourrait être posée pour le TBRV isolé de pomme de terre var. "Anett » au Kenya et qui n'est pas transmis par L. laevicapitatus (KAISER et al., 1978) ou pour le TBRV isolé de betterave à sucre, poivron, pomme de terre et tabac (isolats $\mathrm{N}$ et $\mathrm{N} 1$ ) en Yougoslavie (BUTUROvic et al., 1979).

La spécificité entre le népovirus et un pathotype et l'espèce du nématode a été mentionnée par TRUDGILL et al. (1981).

En Bretagne, la mise en évidence du TBRV chez l'artichaut revêt une importance certaine du fait :

1) de l'existence des grandes surfaces consacrées aux cultures d'artichaut et de pomme de terre,

2) de la présence d'un vecteur potentiel, L. attenuatus, observé dans l'une des 2 cultures et l'abondance d'un hôte spontané $V$. persica qui cohabite avec les 2 cultures.

Ces faits suggèrent que la présence du TBRV chez la pomme de terre cultivée en Bretagne n'est pas à exclure. En effet, ce népovirus observé chez la pomme de terre en Europe de l'Ouest, induirait chez cette plante les mêmes symptômes que ceux provoqués par le Potato Black Ring Virus (PBRV) (SALAZAR \& HARRISON, 1979).

L'observation de ce $4^{\mathrm{e}}$ virus chez l'artichaut contribue à prendre plus en considération les maladies virales dans cette culture et à penser sérieusement à l'organisation d'une mise en place de la régénération de l'artichaut. Celle-ci pourrait s'envisager dans un premier temps, partielle, afin d'en entrevoir les effets. Cependant, à l'évidence, la régénération devrait s'établir après une étude portant, d'une part, sur la nuisibilité et, d'autre part, sur l'épidémiologie des virus présents.

\section{RÉFÉRENCES BIBLIOGRAPHIQUES}

Buturovic D., Grbelja J., Eric Z., 1979. On the geographical distribution of Tomato Black Ring Virus in Yugoslavia. Acta Bot. Croat., 38, $9-12$.

Dalmasso A., Caubel G.. 1966. Répartition des espèces des genres Xiphinema et Longidorus trouvées en France. C. R. Acad. Agric. Fr., 37, 440-446.

Dalmasso A., 1970. Contribution à l'étude des Longidoridae de France (Nematoda, Dorylaimida). Thèse Faculté des Sciences, Univ. Nice.

Harrisson B. D., Mowat W. P., Taylor C. E., 1961. Transmission of a strain of Tomato Biack Ring Virus by Longidorus elongatus (Nematoda). Virology., 14, 480-485.

Kaiser W. J., Bock K. R., Guthrie E. J., Meredith G., 1978. Occurrence of Tomato Black Ring Virus in potato cultivar "Anett" in Kenya. Plant Dis. Rep., 62 (12), 1088-1092.

Migliori A., Lot H., Pecaut P., Duteil M., Rouze-Jouan Joëlle, 1984. Les virus de l'artichaut. I. - Mise en évidence de trois virus dans les cultures françaises d'artichaut. Agronomie, 4 (3), 257-268.
Migliori A., Lot H., Pecaut P., 1984. I es virus de l'artichaut identifiés en Bretagne et dans les régions méridionales. Pépiniéristes Hortic. Maraîchers, 249, 43-47.

Morand J. C., Poutier J. C., 1978. Les taches en anneaux de la laitue, une souche du Tomato Black Ring Virus. Ann. Phytopathol., 10, 101-102.

Murant A. F., 1970. Tomato Black Ring Virus. In C.M.I./A.A.B. Descr. Plant/Viruses, ${ }^{\circ} 38$.

Rana G. L., Martelli G. P., 1983, L'Italia Agricola. Le virosi delle piante ortive. Gennaio - Marzo - Anno 120, 27-38.

Salazar L. F., Harrison B. D., 1979. Potato Black Ring Virus. In C.M.I./A.B.B. Descr. Plant Viruses, $\mathrm{n}^{\circ} 206$.

Trudgill D. L., Brown D. J. F., Robertson W. M., 1981. A comparison of the effectiveness of the four British virus vector species of Longidorus and Xiphinema. Ann. Appl. Biol., 99, 63-70. 